\title{
Reading chest radiographs for pneumoconiosis by computer
}

\author{
J. ROGER JAGOE and KEITH A. PATON \\ Division of Medical Computing, MRC Clinical Research Centre, Northwick Park Hospital, \\ Watford Road, Harrow, Middlesex
}

\begin{abstract}
Jagoe, J. R., and Paton, K. A. (1975). British Journal of Industrial Medicine, 32, 267-272. Reading chest radiographs for pneumoconiosis by computer. Computer programs for measuring simple pneumoconiosis in radiographs are described and assessed. The 36 films studied had been read by 11 skilled human observers and a 'radiological score' of pneumoconiotic severity was therefore available for each film. The computer assigns to each square grid of side $3.6 \mathrm{~mm}$ a measure which reflects the unevenness of the density distribution in that grid. The "computed score' is defined as the mean diversity over all relevant grids in both lung fields. On the set of 36 films the correlation between radiological score and computed score was $\mathbf{0 . 8 8}$. By contrast, the correlation between the score assigned by a single observer and the average of the scores assigned by the other 10 was in the range 0.95 to 0.98 . The program can use the computed score to classify a film into one of the four major International Labour Office (ILO) U/C categories, the success rate of this process being $80 \%$ compared with those quoted by other workers in the range $45 \%-65 \%$. If the films used in this study be typical, then the program described may form the basis of an automatic method for measuring pneumoconiosis in epidemiological work.
\end{abstract}

In epidemiological studies it is sometimes necessary to assess the severity of pneumoconiosis in each of several thousand chest radiographs. Thus National Coal Board observers read some 70000 films a year, many of them in the periodic $x$ ray scheme, which provides a numerical index of relative amounts of radiological change associated with different collieries and thus draws attention to those collieries where radiological change is relatively high. Some 5000 films a year are read in the Department of Employment in examining pneumoconiosis in various industries such as asbestos, pottery, granite, slate, and brewing. The burden of film reading has undoubtedly discouraged other potentially useful surveys from being made.

In each case the question to be answered is not 'Is this chest film abnormal?' but 'How severe is the pneumoconiosis evident in this chest film?' where severity is to be measured according to an inter- nationally agreed set of rules such as the ILO U/C classification scheme (International Labour Office, 1970). Since so many films are involved and the question to be answered is so comparatively well defined, we may reasonably ask whether the assessment can be made by a suitably programmed computer.

\section{Previous work}

Computer diagnosis of pneumoconiosis has been reported by Kruger, Thompson, and Turner (1974) and Hall, Crawford, and Roberts (1975). Both groups see the problem as that of assigning a given film to one of the four major categories.

Hall et al. detect lung fields by program, divide each lung field horizontally into three zones, and take 62 measurements for each zone. They select the best eight of these measurements by stepwise 
TABLE 1

Classification of 38 Films (Hall et al., 1975)

\begin{tabular}{cc|c|c|c|c}
\hline & \multicolumn{4}{|c}{ True category } \\
\cline { 2 - 6 } & & 0 & 1 & 2 & 3 \\
\hline \multirow{3}{*}{ Computed category } & 0 & 8 & 0 & 1 & 0 \\
& 1 & 3 & 8 & 1 & 0 \\
& 2 & 2 & 3 & 3 & 0 \\
& 3 & 0 & 2 & 3 & 4 \\
\hline
\end{tabular}

discriminant analysis (Dixon, 1968) and use a linear discriminant function to classify each individual zone. Finally, they define rules for combining the results for the six zones in order to classify the film as a whole. Their main result on 38 films is shown in Table 1.

Kruger et al. use two methods. In one, an optical computer is used to calculate the Fourier spectrum over a circle of diameter $63.5 \mathrm{~mm}$ placed by hand in the lung field, and the normalized energy in the separate annuli of the Fourier plane are discriminating variables. In the other, they calculate some 80 textural measurements, based on the point-to-point variations in density, on squares of side $1 \mathrm{~cm}$ placed by hand in the inter-rib spaces. In both methods, they select the best measurements by stepwise discriminant analysis and use a linear discriminant function to classify an elementary region (circle in the first case, square in the second). In the first case, this serves to classify the film as a whole since there is one circle per film; in the second case, the results from all the little squares are combined to give a result for the whole film. Their main results are by Fourier analysis on 141 films (Table 2) and by textural analysis on 95 films (Table 3).

Since both groups define 'true category' for a film as the majority verdict of six readers each using the four-point scale, it follows that they see the problem as one of assigning a given film to one of four classes of pneumoconiotic severity and that the quality of their method is measured as a percentage of films correctly categorized. Since the amount of simple

TABLE 2

Classification of 141 Films (Kruger et al., 1974)

\begin{tabular}{lr|r|r|r|r}
\hline & \multicolumn{4}{|c}{ True category } \\
\cline { 2 - 6 } & & 0 & 1 & 2 & 3 \\
\hline \multirow{3}{*}{ Computed category } & 0 & 29 & 5 & 1 & 1 \\
& 1 & 8 & 14 & 6 & 10 \\
& 2 & 0 & 3 & 10 & 11 \\
& 3 & 2 & 11 & 13 & 17 \\
\hline
\end{tabular}

TABLE 3

Classification of 95 Films (Kruger et al., 1974)

\begin{tabular}{cc|r|r|r|r}
\hline & & \multicolumn{3}{|c}{ True category } \\
\cline { 3 - 6 } & & 0 & 1 & 2 & 3 \\
\hline \multirow{3}{*}{ Computed category } & 0 & 30 & 1 & 0 & 1 \\
& 1 & 1 & 5 & 3 & 3 \\
& 2 & 0 & 7 & 15 & 13 \\
& 3 & 0 & 2 & 1 & 13 \\
\hline
\end{tabular}

pneumoconiosis shown by a film is in principle a continuous variable grouped by observers into 12 classes, it is natural for us to see the problem as one of assigning a computed score to a film and to measure the quality of our method as the correlation between computed score and radiological score, defined as the arithmetic mean of the readings obtained by a number of readers on the 12-point scale.

\section{Materials and methods}

The films used were chosen out of a set of 394 films from many different sources used by Rossiter (1972) in his study of the relation between radiological appearance and the dust content of coalworkers' lungs. Each film had been read by 11 trained human observers using the 12-point National Coal Board elaboration (Liddell, 1963) of the four-point ILO scale (International Labour Office, 1959). Each film had been taken within three years of the death of the subject and the dust content of the lungs had been measured after death, thus providing a dust score for that film. Thirty-six films were chosen for analysis by computer, nine each from the lower end of category 0 , the middles of categories 1 and 2, and the upper end of category 3. The films chosen were those showing most agreement among the 11 readers. No attempt was made to select films of good quality; the films chosen were of radiographic quality typically encountered in practice.

In order to define an average score for each film the 12 points $(0 /-, 0 / 0,0 / 1,1 / 0,1 / 1,1 / 2,2 / 1,2 / 2,2 / 3,3 / 2$, $3 / 3,3 / 4$ ) of the scale were replaced by the numbers 1 to 12 . If $\mathrm{srt}_{\mathrm{r}}$ denotes the numerical score thus derived from the scale point assigned to film $f$ by reader $r$, then the radiological score is defined as $\sum_{\mathrm{r}=1}^{11} \mathrm{sr} / 11$. Note that this score is not the same as that derived by Rossiter (1972). Our task is to write a program which derives from the chest films a computed score (cs) that agrees well with radiological score.

The process may be expressed in three steps. First, the film is scanned to provide a density matrix on which the computer can operate. Secondly, the matrix is analysed to detect the lung field. Thirdly, the relevant part of the matrix is analysed to measure pneumoconiotic severity. This paper is mainly about the third step, but for completeness we briefly summarize the first two steps.

Since the scanner available cannot digitize the full size 
chest film, it is necessary to reduce the original film by four, producing a version about $10 \mathrm{~cm}$ square which is digitized at $0.3 \mathrm{~mm}$ intervals to yield a matrix $D$ of integral values where $d_{i j}$ is approximately proportional to the mean density in a square of side 0.3 with centre $(3 \mathrm{i} / 10,3 \mathrm{j} / 10)$, all distances being in millimetres. Each value is in the range 0,255 , where 255 corresponds to optical density $1 \cdot 8$. The effective sampling interval as measured in the original film is thus $1.2 \mathrm{~mm}$.

An approximation to the lung field is defined as follows: individual edge points are detected as abrupt changes in density in single vertical lines. The set is then edited to yield a succession of alternately rising and falling density steps. Edge points are linked between adjacent vertical lines to form rib edges, the linking process allowing for missing values. The lung field is then defined as the envelope of all significant rib edges.

It is convenient for later analysis to express the computed lung field in terms of horizontal slices each the width of the lung field and of height about that of an interrib space, as shown in Fig. 1. The discrepancies between the true lung field and that detected by the computer are unimportant, since the characteristic opacities of pneumoconiosis are rarely confined to the peripheral portion of lung field missed by the computer.

In other investigations, it might be necessary to use a more accurate technique for finding the lung fields, such as the elegant boundary following technique (Chien and $\mathrm{Fu}, 1974$ ).

We now consider the third step, the measurement of pneumoconiotic severity. Each of the slices referred to above is covered with a non-overlapping set of nine squares (Fig. 2) where the circles denote density values. We shall refer to such a set as a 'grid'. Every grid corresponds to a piece of the small film $0.9 \mathrm{~mm}$ square and hence to a piece of the full-size film $3.6 \mathrm{~mm}$ square, taking account of the four-to-one reduction. Hence a quantity

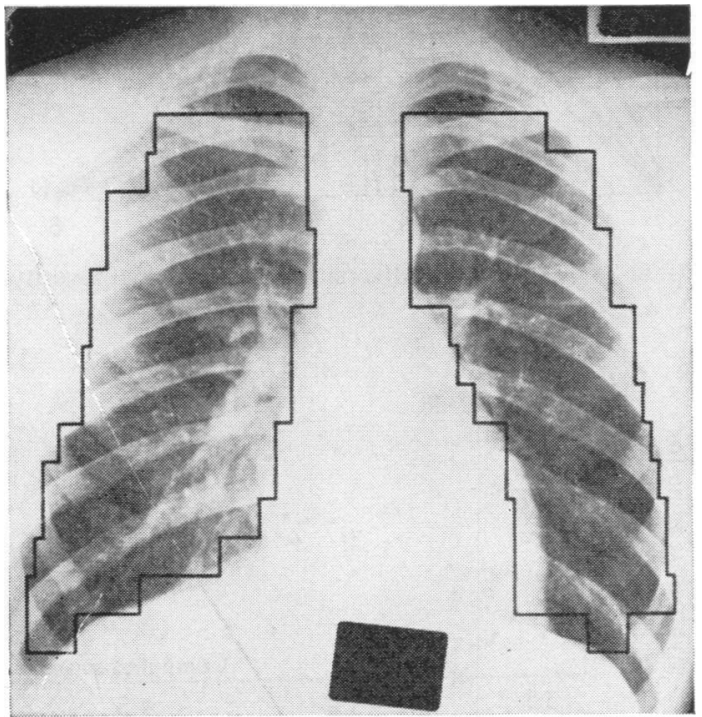

FIG. 1. Lung field detected by computer.

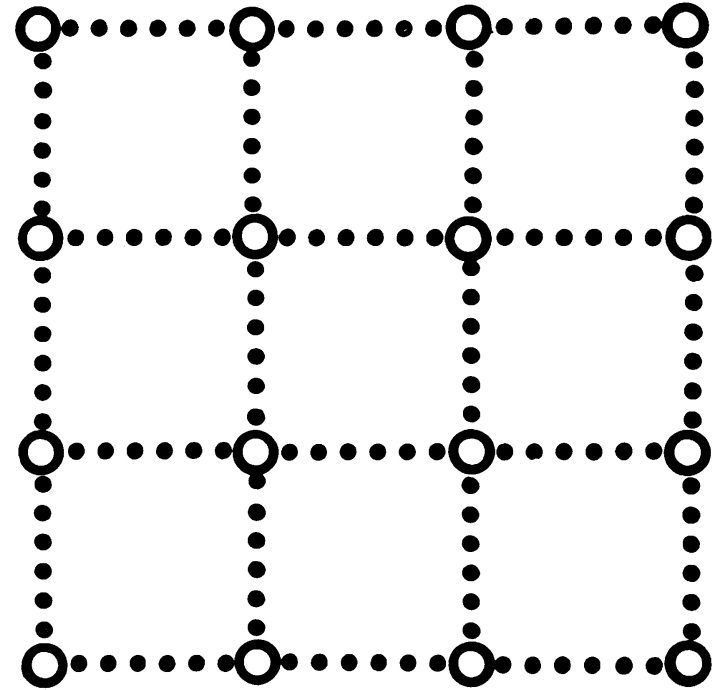

FIG. 2. Array of squares in a grid.

measured by the computer on the evidence of the 16 density values shown above is a property of the corresponding piece of film $3.6 \mathrm{~mm}$ square.

In a single square, consider the increase in density as we cross the square in a given direction. Thus, suppose the densities are A, B, C, D as shown in Fig. 3, then the rates of increase in the eight principal directions are as shown in Fig. 4. For each square we select the direction with the fastest increase in density, thus obtaining a situation such as that in Fig. 5.

In the case of a tie, the single winning direction is chosen as the first occurring winning direction in Fig. 4. The value of this fastest increase is known as the "gradient of density'.

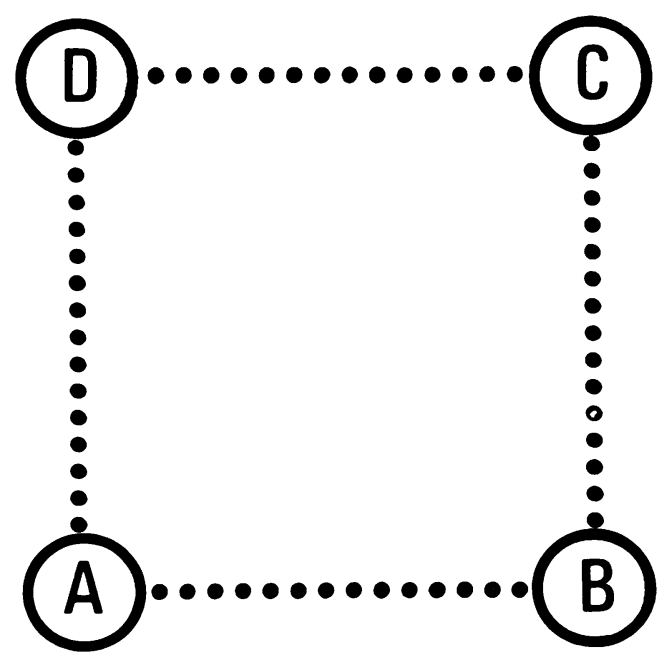

FIG. 3. Densities in a square. 


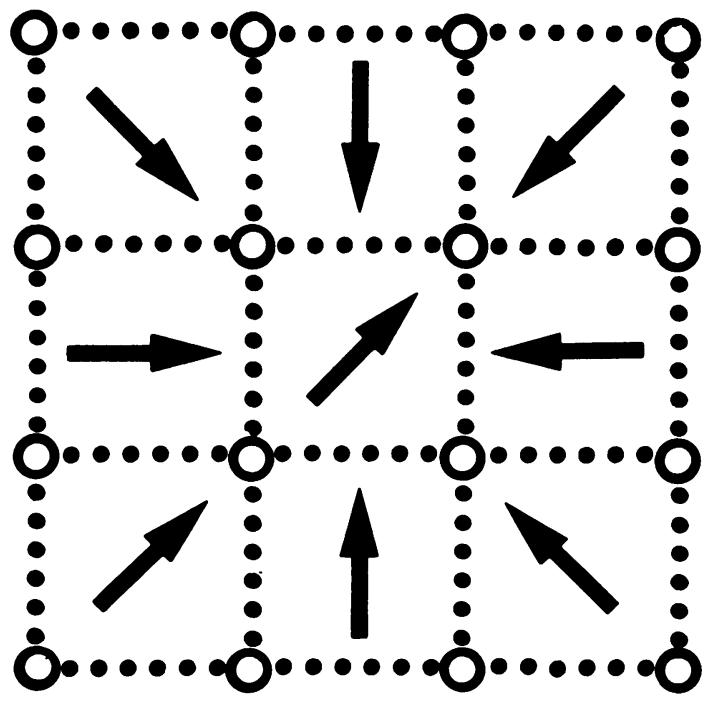

Fig. 5. Array of gradient direction.

At this point we may discard the grid for one of two reasons.

First, if the arithmetic mean of the 16 density values is less than the median density for the current slice of lung field, we assume that the grid is situated over a rib rather than an inter-rib space. Since we wish to examine only the inter-rib spaces, such a grid is discarded.
Secondly, if the gradient of density in the central square is less than the median gradient for the current slice, the grid is discarded. In this way, we try to confine our attention to those regions where the density is changing more rapidly than is usual.

Both discarding rules are justified by experience; we discover that the program performs more accurately with them than without them. It is worth noting, however, that the naive assumption behind the first rule has been found to be invalid. Discarding a grid if the average density is less than the median density in the slice might be expected to restrict the search to the inter-rib spaces but experience shows that it does not. We have not yet examined the situation in detail but our impression is that some $30 \%$ of all surviving grids lie at least partly out of the inter-rib spaces.

Suppose the grid has not been discarded. We assume that on smooth or featureless areas the arrows in the nine squares will point in more or less the same directions and that on areas containing opacities the arrows will point in many different directions. We therefore record for each surviving grid the number of different directions. Let us define this number as the 'diversity' of the grid.

The values of diversity on surviving grids may be summarized in a frequency distribution. Figure 6 shows

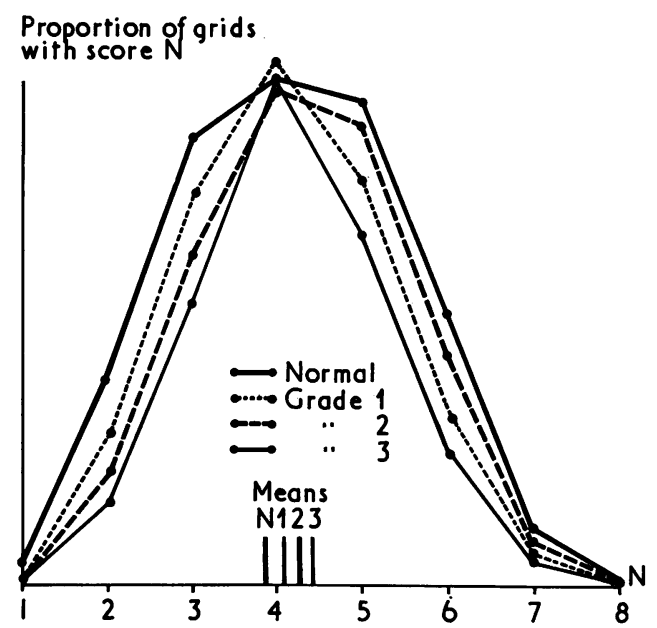

FIG. 6. Distribution of diversity by radiological severity.

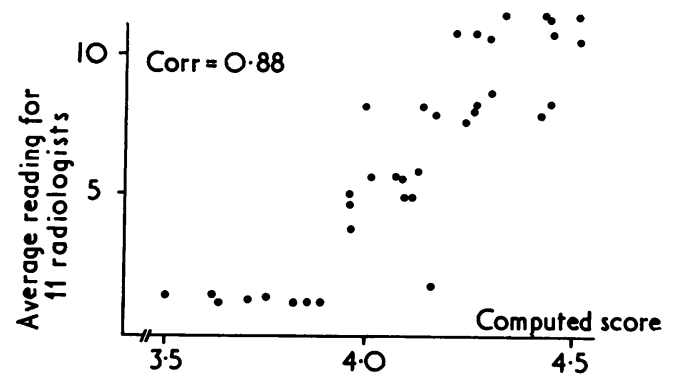

Fig. 7. Computed score and radiological score. 
TABLE 4

INTER-OBSERVER CORRELATIONS

\begin{tabular}{c|c}
\hline Observer & Correlation with remainder of group \\
\hline 1 & 0.95 \\
2 & 0.96 \\
3 & 0.96 \\
4 & 0.95 \\
5 & 0.96 \\
6 & 0.96 \\
7 & 0.96 \\
8 & 0.96 \\
9 & 0.95 \\
10 & 0.98 \\
11 & 0.97 \\
\hline
\end{tabular}

[The correlation between the computer and the group as a whole was $0 \cdot 88$ ]

typical distributions for four grades of pneumoconiosis. Higher grades of pneumoconiosis have more high values of diversity. We therefore define 'computed score' as the mean diversity over surviving grids in both lung fields. Figure 7 shows the distribution of computed score and radiological score on the set of 36 films where the correlation between the two is $\mathbf{0 \cdot 8 8}$. Table 4 compares this with the correlation between the scores assigned by an individual observer and the average of the scores assigned by the other 10 .

\section{Discussion}

There are two main reasons why we should expect the correlation between computed score and radiological score to be lower than that between human observers.

First, the films chosen were those on which the human observers agreed best. As shown in Table 5, the agreement among human observers was better on the chosen set of 36 films than on the whole set of 394 films. This introduces bias in favour of the human observers.

Secondly, the computer adopts a naive approach to the problem; it does not attempt to discard blood vessels from the analysis, although we know from observation that diversity is high on blood vessels.

TABLE 5

VARIANCE BETWEEN OBSERVERS

\begin{tabular}{c|c|c}
\hline Category & Complete set of 394 & Selected 36 \\
\hline 0 & $2 \cdot 83$ & $2 \cdot 30$ \\
1 & $10 \cdot 66$ & $6 \cdot 37$ \\
2 & $11 \cdot 54$ & $8 \cdot 43$ \\
3 & 6.88 & $6 \cdot 09$ \\
\hline
\end{tabular}

The scoring system is a modification of that suggested by Rossiter (1972)
Indeed, the one film labelled as category 2 by computer but normal by human observers is one with very well-defined blood vessels. Thus, if blood vessels could be traced and rejected during the analysis, we might reasonably expect computer performance to improve. It is perhaps worth noting also that the computer classification is in principle perfectly repeatable, whereas that of a human observer is not very much better than the agreement between two observers.

In comparing our method with those of others, we note that both Hall et al. (1975) and Kruger et al. (1974) measure several variables and use linear discriminant analysis to carry out the assignment. Since we need only one variable, we can adopt their classification rule in the following simple form.

To assign film $\mathrm{f}$, consider only the $\mathbf{3 5}$ films other than $f$. Let $m_{i}$ denote the mean computed score for the films agreed by the panel of 11 observers to be in category $i$ and let the boundary between categories $i, i+1$ be defined as $\left(m_{i}+m_{i+1}\right) / 2$. Comparison of the computed score for film $f$ with these boundaries yields the computed category. Table 6 shows categories assigned by the computer and by the panel of 11 observers.

TABLE 6

Classification of 36 Films by our Method

\begin{tabular}{ll|l|l|l|l}
\hline & \multicolumn{4}{|c}{ True category } \\
\cline { 3 - 6 } & & 0 & 1 & 2 & 3 \\
\hline \multirow{3}{*}{ Computed category } & 0 & 8 & 0 & 0 & 0 \\
& 1 & 0 & 9 & 2 & 0 \\
& 2 & 1 & 0 & 6 & 3 \\
& 3 & 0 & 0 & 1 & 6 \\
\hline
\end{tabular}

Our results, together with those of other computer methods, are set out in Table 7 . The accuracy is the percentage of films assigned to the correct category. Direct comparison is difficult, since the different methods have been tested on different sets of films. However, if the sets used be representative of those

TABLE 7

ACCURACY OF Different COMPUTER MethodS

\begin{tabular}{l|rc|c}
\hline \multicolumn{1}{c|}{ Method } & \multicolumn{2}{|c|}{ No. of } & Accuracy \\
& films & variables & $\%$ \\
\hline This paper & 36 & 1 & $80 \cdot 5$ \\
Kruger et al., 1974 (texture) & 95 & 7 & $65 \cdot 5$ \\
Hall et al., 1975 & 38 & 8 & $60 \cdot 5$ \\
Kruger et al., 1974 (Fourier) & 141 & 6 & $49 \cdot 6$ \\
\hline
\end{tabular}


encountered in practice, then we may reasonably conclude that our results are superior to those of the other authors.

As shown in Table 7, both groups employ several variables. By contrast, the diversity function is a good measure of pneumoconiotic severity on its own and we therefore find that multivariate analysis is unnecessary.

If the method we have described is to form part of a system for measuring the severity of pneumoconiosis in humans, then in assessing it we must take account of the other parts of such a system. In our case, the system may be expressed in four steps.

1. Take chest film of subject.

2a. Reduce chest film fourfold to obtain small film.

b. Digitize small film, that is, convert it to computer readable form.

3. Calculate computed score.

As explained earlier, the reduction in step $2 \mathrm{a}$ is necessary because our densitometer cannot deal with full-size films. In an operational system, steps $2 a, 2 b$ would be replaced by 2 . Digitize chest film.

Now, the computed score for a subject may be affected by several things other than pneumoconiosis. Differences in radiographic technique can produce films of markedly different appearance from the same subject. Differences in photographic technique can produce different small films from the same large film. Differences in digitization technique can produce different computer readable versions from the same small film. Work is in progress to investigate the relation between variations of this kind and variations in computed score.
We acknowledge the valuable assistance of members of the MRC Pneumoconiosis Unit and the Atlas Computer Laboratory and should also like to thank Dr C. C. Spicer for helpful advice.

\section{References}

Chien, Y. P. and Fu, K. S. (1974). A decision function method for boundary detection. Computer Graphics and Image Processing, 3, 125-1240.

Dixon, W. J. (1968). BMD Biomedical Computer Programs, University of California Press, 214a-214t.

Hall, E. L., Crawford, W. O., Jr., and Roberts, F. E. (1975). Computer Classification of Pneumoconiosis in Radiographs of Coal Workers. Institute of Electronic and Electrical Engineers Transactions on Biomedical Engineering, Vol. BME-22, 518-528.

International Labour Office (1970). International Classification of Radiographs of Pneumoconioses (Review 1968), Occupational Safety and Health, Series No. 22, ILO, Geneva.

Kruger, R. P., Thompson, W. B., and Turner, A. F. (1974). Computer Diagnosis of Pneumoconiosis, Institute of Electronic and Electrical Engineers Transactions on Systems, Man and Cybernetics, SMC-4, 1, 40-49.

Liddell, F. D. K. (1963). An experiment in film reading. British Journal of Industrial Medicine, 20, 300-312.

Rossiter, C. E. (1972). Relation between content and composition of coal workers' lungs and radiological appearances. British Journal of Industrial Medicine, 29, 31-44.

Paper received for publication 28 November 1974

Paper accepted for publication 21 February 1975 